\title{
HBV Infection Statuses Indicate Different Risks of Synchronous and Metastasis Liver Metastasis in Colorectal Cancer
}

\section{Jiaming Zhou}

Sixth Affiliated Hospital of Sun Yat-sen University

\section{Pinzhu Huang}

Sixth Affiliated Hospital of Sun Yat-sen University

\section{Xiaoyan Guo}

Third Affiliated Hospital of Sun Yat-sen University

\section{Shuyun Tan}

Sixth Affiliated Hospital of Sun Yat-sen University

\section{Rongwan Lin}

Sixth Affiliated Hospital of Sun Yat-sen University

Huanmiao Zhan

Sixth Affiliated Hospital of Sun Yat-sen University

\section{Xiaofeng Wu}

Sixth Affiliated Hospital of Sun Yat-sen University

\section{Shaoyong Peng}

Sixth Affiliated Hospital of Sun Yat-sen University

\section{Tuoyang Li}

Sixth Affiliated Hospital of Sun Yat-sen University

\section{Mingzhe Huang}

Sixth Affiliated Hospital of Sun Yat-sen University

\section{Yuanlv Xiao}

Sixth Affiliated Hospital of Sun Yat-sen University

Jianping Wang

Sixth Affiliated Hospital of Sun Yat-sen University

Meijin Huang ( $\nabla$ hmjin@mail.sysu.edu.cn )

Sixth Affiliated Hospital of Sun Yat-sen University

\section{Research Article}

Keywords: colorectal cancer, liver metastasis, hepatitis B virus, HBV infection, HBsAg 
Posted Date: March 15th, 2021

DOI: https://doi.org/10.21203/rs.3.rs-285576/v1

License: (c) (i) This work is licensed under a Creative Commons Attribution 4.0 International License. Read Full License 


\section{Abstract}

Background: Previous studies on the effect of HBV infection on CRLM are contrary. This study identified more specific and different impacts of HBV on CRLM.

Methods: A total of $3132 \mathrm{CRC}$ patients were analyzed retrospectively and followed up for five years. All patients were divided into three groups: group $\mathrm{A}, \mathrm{HBsAg}$ positive with $\mathrm{HBV}$ infection; group $\mathrm{B}, \mathrm{HBsAg}$ negative with HBV infection; and group C, no HBV infection. The risk factors for SYN-CRLM, MET-CRLM, 5 -year OS, and LDFS were analyzed.

Results: A total of 829 patients had CRLM. SYN-CRLM was found in 425 patients. The incidence of SYNCRLM was $16.95 \%, 11.60 \%$ and $13.50 \%(P<0.03)$ in groups $A, B$, and $C$, respectively. HBsAg-positive HBV infection increased the risk of SYN-CRLM $(P<0.01)$, with a worse prognosis in group $A(P<0.05)$. METCRLM was found in 404 patients. The incidence of MET-CRLM in groups $A, B$, and $C$, was $16.51 \%, 11.53 \%$ and $16.50 \%(P=0.02)$. HBsAg-negative HBV infection decreased the incidence of MET-CRLM $(P=0.02)$ with a better 5-year LDFS $(P=0.01)$, but was not related to 5-year OS $(P=0.15)$.

Conclusion: HBsAg positivity infection increased the risk of SYN-CRLM with poor prognosis. HBsAgnegative infection reduced the risk of MET-CRLM with better LDFS after surgery.

\section{Introduction}

In recent years, the global incidence of colorectal cancer (CRC) has continued to rise. CRC is the third most common malignant tumor with the second-highest mortality rate ${ }^{1}$. In China, the incidence and mortality of $\mathrm{CRC}$ are ranked fourth and fifth ${ }^{2}$. The majority of CRC patients who died had distant metastasis, and liver metastasis was the most common. Synchronous CRC liver metastasis (SYN-CRLM) was shown in $14-20 \%$ of CRC patients at the time of diagnosis ${ }^{3-5}$. In patients without SYN-CRLM, nearly $17 \%$ of them suffered from metachronous CRC liver metastasis (MET-CRLM), even after resection of the primary tumor ${ }^{6,7}$. According to statistics, the SYN-CRLM rate of colorectal cancer in China is about 25$30 \%$, and the MET-CRLM rate is about $12-21 \%$.

Hepatitis B virus (HBV) infection is the most common chronic liver disease in humans. It is estimated that approximately 2 billion people worldwide have been infected with $\mathrm{HBV}$, and more than 350 million people have chronic hepatitis $\mathrm{B}(\mathrm{CHB})^{8}$. Approximately 120 million people in China are chronic HBV carriers, accounting for approximately one-third of the world's total population, and 30 million of them have HBV virus replication throughout their life ${ }^{9,10}$. Hepatitis $B$ is one of the causes of hepatocellular carcinoma, increasing the risk of intrahepatic metastasis ${ }^{11-13}$. In addition, it has been reported that HBV infection also increases the risk of liver metastasis in pancreatic cancer, B-cell lymphoma, and other malignant tumors ${ }^{14-16}$. 
Several previous studies have reported the risk of HBV for CRC liver metastasis, but there are two different opinions. Utsunomiya et al. suggested that the risk of CRLM is lower in patients with HBV and HCV infections ${ }^{17}$. Song et al. suggested that HBV replication can reduce the incidence of CRLM ${ }^{18}$. Augustin et al. and Cai et al. reported in a meta-analysis that the risk of liver metastasis in CRC patients with CHB was reduced ${ }^{19,20}$. Zhao et al. suggested that HBV infection is a good predictor of prognosis after liver metastasis resection ${ }^{21}$. However, in recent years, scholars have raised different objections. Huo et al. indicated that hepatitis B virus surface antigen ( $\mathrm{HBsAg}$ ) positivity is an independent risk factor for SYN$\mathrm{CRLM}^{22}$. All of the above studies have some limitations. There are no analyses of CRLM in different periods based on different HBV results and HBV infection statuses. At present, there is still a lack of large samples, comprehensive retrospective studies, and studies with support from laboratory data. Therefore, this study reviewed the data of 3040 patients treated at the Sixth Affiliated Hospital of Sun Yat-sen University from 2013 to 2015 and analyzed the relationship between HBV infection and CRLM.

\section{Patients And Methods}

\section{Patients}

From January 1,2013 , to December 31,2015 , a total of 3914 newly admitted and confirmed CRC patients were analyzed retrospectively. The inclusion criteria were as follows: (1) pathological diagnosis of colorectal adenocarcinoma; (2) no current or previous history of other malignant tumors; (3) no viral coinfection of the liver, except HBV; and (4) absence of drug hepatitis, alcoholic liver disease, fatty liver disease, autoimmune liver disease, and pregnancy with CHB. Patients who died within 30 days after surgery were excluded. Patients without hepatitis B virus serological marker (HBVM) or liver examination results at the first diagnosis of CRC were excluded. A total of 3132 patients were enrolled in this study. The medical ethics committee of the Sixth Affiliated Hospital of Sun Yat-sen University approved this study (Ethics approval number 2020ZSLYEC-101). Research was performed in accordance with the relevant guidelines and regulations. All participants provided written informed consent according to the declaration of Helsinki. See Fig1.

\section{Data collection}

The data included sex, age, tumor location, differentiation degree, HBVM results at first admission, carcinoembryonic antigen (CEA), carbohydrate antigen 19-9 (CA19-9), aspartate aminotransferase (AST), alanine aminotransferase (ALT), fibrosis index based on the four factors (FIB-4), chemotherapy (including neoadjuvant or adjuvant chemotherapy), Tumor, Node, Metastasis (TNM) stage (according to American Joint Committee on Cancer (AJCC), version 7 of CRC), liver disease-free survival (LDFS) and overall survival (OS) during follow-up.

\section{Judgment of CRLM}


There are variable definitions of synchronous and metachronous CRLM reported in the literature. According to the statements from the Expert Group on OncoSurgery management of Liver Metastases group (EGOSLIM), SYN-CRLM should be termed "synchronously detected liver metastases" 23 . We diagnosed the intrahepatic nodule as SYN-CRLM at the first diagnosis of CRC and MET-CRLM at any time after that.

In this study, for the intrahepatic nodule, after the first diagnosis of CRC, we examined the patient's radiology images, detected the serum CEA and alpha-fetoprotein (AFP) levels, and performed ultrasoundguided biopsy and pathological diagnosis when necessary. After excluding primary liver cancer, hemangioma, and liver cyst, all the results were judged by two radiologists who had more than 5 years of experience in specifically diagnosing CRLM.

\section{Judgment of HBV infection}

All patients were tested for HBV using HBVM (five items of hepatitis B) at the first diagnosis of CRC. According to the results of HBsAg, hepatitis B surface antibody (anti-HBs), hepatitis B e antigen (HBeAg), hepatitis $B$ e antibody (anti-HBe), and hepatitis B core antibody (anti-HBc). We divided patients into three groups: groups A, B, and C. Group A included patients with HBsAg-positive HBV infection. Group B included patients with $\mathrm{HBsAg}$-negative $\mathrm{HBV}$ infection who were positive for $\mathrm{HBeAg}$, anti-HBe, and antiHBc. Group C included patients who were negative for HBVM or were only anti-HBs-positive.

\section{Follow-up}

The time between the first diagnosis of CRC and the diagnosis of MET-CRLM was defined as LDFS, and the time between the first diagnosis of CRC and death was defined as OS. CT or B-ultrasound examinations were performed every 6-12 months after the operation. If abnormal nodules in the liver were found, the diagnosis of CRLM was made by contrast-enhanced ultrasonography or MRI. If necessary, a biopsy was performed for pathological diagnosis. The follow-up office of the Sixth Affiliated Hospital of Sun Yat-sen University followed up all the patients. We collected and recorded the follow-up data through the patient's return visit. For the patients who are examined in a local hospital due to living in a remote area, if there was suspected tumor recurrence or progress, they were required to return to our hospital for further diagnosis.

\section{Statistical analysis}

Categorical variables are shown as the number and percentage of patients. For binary variables, baseline categorical clinical parameters were compared by $\chi^{2}$-test or Fisher's exact test, and numerical values were compared using Student's t-test. Significant risk factors for SYN-CRLM and MET-CRLM were analyzed by logistic regression analysis. A log-rank test was used to determine OS and LDFS in each group. The 
hazard ratio $(\mathrm{HR})$ and the corresponding $95 \%$ confidence interval $(\mathrm{Cl})$ were estimated with a mixed effects Cox regression model. Statistical analysis was performed by SPSS 22 (IBM company) and GraphPad Prism 8. A two-tailed P-value less than 0.05 was considered a significant difference.

\section{Results}

\section{Baseline characteristics}

From January 1, 2013, to December 31, 2015, 3914 CRC patients were confirmed of having CRLM in our hospital. 782 patients were excluded because of the lack of HBVM results and the inability to evaluate preoperative liver metastasis. A total of 3132 patients were included in final analysis, which included 1922 men and 1210 females. The median age was 56 years ( \pm 11.17 , range from 17 to 95$)$. There were 1526 patients with rectal cancer and 1606 patients with colon cancer. According to the HBVM results, patients were divided into group A, B or C. Group A was the HBsAg-positive group, with a total of 413 (13.19\%) patients. Group B was the HBsAg-negative infection group, with a total of 638 (20.37) patients. Group C was the non-infection group, with a total of 2081 (66.44\%) patients. 2893 patients received surgery of primary tumor resection during their treatment, while the other 239 did not.

SYN-CRLM were found in 425 (13.57\%) patients at their first diagnosis, while the other 2707 were not. In those who without SYN-CRLM, MET-CRLM happened in 404 (12.90\%) patients after surgery, while CRLM was not found in the remaining 2211 (70.59\%) patients during follow-up. 92 patients did not have surgery and lost connection. See Table 1

\section{Differences between HBV infection groups}

The statistical results showed that there was no difference in tumor location, tumor differentiation, $T$ stage, $\mathrm{N}$ stage, CEA and CA19-9 in different HBV infection groups. In the male proportion, there was a significant difference between group $A(62.95 \%)$, group $B(65.20 \%)$ and group $C(59.88 \%)(P=0.04)$. The proportion of patients younger than 56 years old in group $A(56.90 \%)$ was higher than that in group $B$ $(43.89 \%)$ and group C (45.65\%) $(P<0.01)$. The abnormal proportion of AST $(P<0.01)$, ALT $(P<0.01)$ and FIB-4 $(P<0.01)$ in group A was significantly higher than that in group $B$ and group $C$, with statistical differences. See Table 2

Among the 3132 patients with CRC (before operation), the incidence of SYN-CRLM in group A, group B and group C was 16.95\% (70 / 413), 11.60\% (74 / 638) and 13.50 (281 / 1800), respectively, with statistical difference $(P=0.05)$. See Table 3. Of the 2707 patients without SYN-CRLM, 92 were lost because of refusing surgery or transferring to other hospitals. 2615 patients underwent primary tumor resection and were followed up. The incidence of MET-CRLM in group A, B and C was $16.51 \%$ (54 / 327), $11.53 \%$ (64 / 555) and 16.50\% (286/ 1733), respectively, with statistical difference $(P=0.02)$. See Table 4. 


\section{Risk factors for SYN-CRLM}

In this study, a total of 425 people was found to have SYN-CRLM at the first diagnosis of CRC before surgery. Significant differences in tumor location, pathology differentiation, T stage, $N$ stage, HBV infection, CEA, CA19-9, AST, ALT between patients with and without SYN-CRLM were found. There were no significant differences in sex, age or FIB-4 among the SYN-CRLM group and the non-SYN-CRLM groups. In multivariate analysis, high or moderate differentiation ( $P=0.01, H R=0.62(0.44-0.87)$ ) was an independent protective factor for SYN-CRLM. The independent risk factors for SYN-CRLM were T stage of 3 or $4(P<0.01, \mathrm{HR}=3.94(2.08-7.48)), \mathrm{N}$ stage of 1 or $2(P<0.01, \mathrm{HR}=1.83(1.38-2.46)), \mathrm{CEA}>5 \mathrm{ng} / \mathrm{ml}$ $(P<0.01, \mathrm{HR}=3.85$ (2.84-5.22)), CA19-9>37 U/ml $(P<0.01, \mathrm{HR}=2.18$ (1.59-2.98)), AST $>40(P<0.01, \mathrm{HR}=3.85$ (2.84-5.22)), and ALT>40 U/L ( $\mathrm{P}<0.01), \mathrm{HR}=2.15$ (1.49-3.10)). In addition, HBsAg positive was an independent risk factor for SYN-CRLM $(P<0.01)$. Compared with HBsAg positive group, the relative risk of SYN-CRLM in HBsAg negative group was 0.19 (0.11-0.34), while that of non-infection group was 0.29 (0.18-0.47). See Table 5.

\section{Risk factors for MET-CRLM}

After excluding SYN-CRLM, 92 patients were lost because of refusal further treatment or transfer to other hospital. A total of 2615 patients had surgery for primary tumor resection and were followed up; of these patients, 404 developed MET-CRLM, accounting for $12.90 \%$. In patients with MET-CRLM and those without MET-CRLM, there were no differences in sex, age, AST level, ALT level, or FIB-4 level. There were significant differences in the location of the primary tumor, degree of tissue differentiation, $\mathrm{T}$ stage, $\mathrm{N}$ stage, M stage, CEA level, CA19-9 level and chemotherapy $(P<0.01)$.

In the Cox regression analysis, results showed that better pathological differentiation $(P=0.04, H R=0.77$ (0.61-0.99)) were independent protective factors. The presence of extrahepatic organ metastasis $(P<0.01$, $H R=10.12$ (7.90-12.95)), initial CA19-9 level at the first diagnosis of $C R C(P<0.01, H R=1.50$ (1.19-1.89)) and chemotherapy $(P<0.01, \mathrm{HR}=3.11$ (1.91-5.04)) were independent risk factors.

There were also differences in the HBV infection groups $(P=0.02)$. What was different from SYN-CRLM, we found that HBsAg negative infection in group $B$ was an independent protective factor to MET-CRLM (relative to group $A, P=0.04, H R=1.47$ (1.02-2.10) and group $C, P<0.01, H R=1.49$ (1.14-1.95)). See Table 6.

\section{5-year OS and LDFS between different HBV infection groups}

In this study, the follow-up period ended on January 1, 2020. A total of 2893 patients were followed up with a median follow-up time of 43 months (range from 0-73). 239 patients were lost. A total of 587 people died, 2306 survived during the follow-up. The 5-year survival rate was $79.71 \%$. The mortality of patients in groups A, B, and C was 24.87\% (93/374), 19.26\% (115/597), and 19.72\% (379/1922), 
respectively. There was a significant difference in the 5-year OS rate among the three groups $(P=0.05)$. See Fig2.

During the follow-up, in 2615 patients who had surgery and were without SYN-CRLM, 404 MET-CRLM patients were found, and the 5 -year LDFS was $84.55 \%$ (2211/2615). The MET-CRLM rates of groups A, B, and $C$ were $16.51 \%(54 / 327), 11.53 \%(64 / 555)$, and $16.50 \%$ (286/1733), respectively. There was a significant difference in the 5-year LDFS $(P=0.01)$. See Fig3. In 2615 patients without SYN-CRLM, 450 died, and the OS rate was $82.79 \%$. There were no significant differences among the groups $(P=0.15)$. See Fig4.

\section{Discussion}

There have been several previous studies on the relationship between HBV and CRLM, but the conclusions are contradictory. According to Qi et al., the risk of MET-CRLM in patients with CHB is $14.2 \%$, which is significantly lower than that in control group $(28.2 \%)^{24}$. Li et al. suggested that the chance of CRLM in patients with CHB or chronic Hepatitis C (CHC) decreased $3.2 \%$, comparing with $9.4 \%$ in no infected group ${ }^{25}$. Wang et al. showed that the risk of CRLM in patients with $\mathrm{CHB}$ or $\mathrm{CHC}$ was $2.86 \%$, significantly lower than $16.9 \%$ in no infected group ${ }^{26}$. On the contrary, Huo et al. indicated that patients who are HBsAg-positive are more likely to have SYN-CRLM ${ }^{22}$. None of the above studies considered that HBV infection might have different effects on CRLM before and after surgery, and these studies did not analyze the effects caused by different HBV infection statuses independently. We believe that is the reason why they have different conclusions.

In this study, the incidence of CRLM was $26.47 \%$ (829/3040), and the incidence in groups A, B and C were $30.02 \%$ (124/413), 21.63\% (138/629) and 27.25\% (567/2081), respectively. There were significant differences among the three groups $(P<0.01)$, suggesting that three different $\mathrm{HBV}$ infection statuses have different effects on CRLM.

According to the examination at the first diagnosis of CRC, SYN-CRLM was found in 425 patients, accounting for $13.57 \%, 2707$ patients did not have SYN-CRLM. The incidence of SYN-CRLM in group B $(\mathrm{P}<0.01, \mathrm{HR}=0.19,95 \% \mathrm{Cl}(0.10-0.33))$ and group $\mathrm{C}(\mathrm{P}<0.01, \mathrm{HR}=0.28,95 \% \mathrm{Cl}(0.17-0.46))$ was significantly lower compared to group $A$. This indicates that the active replication of HBV may increase the risk of SYN-CRLM, and this result is the same as that found by Huo et al. ${ }^{22}$. Abnormal elevation of AST and ALT were independent risk factors for SYN-CRLM, suggesting that SYN-CRLM may be related to HBV-induced hepatocyte damage.

After resection of primary tumor in 2615 patients, MET-CRLM was found in 404 patients $(15.45 \%)$ during the follow-up. However, different from the effect on SYN-CRLM, HBV infection (including groups $A$ and B) was an independent protective factor relative to group $C$ in the Cox regression analysis. The 5-year LDFS rate of group $B$ was $88.47 \%$, which was significantly better than that of group $A(83.49 \%)$ and group $C$ (83.50\%) $(P=0.01)$. This conclusion is similar to that from many previous studies ${ }^{17-20}$. This result 
suggested that the liver's immune status may reduce the risk of CRLM in patients who do not have HBV replication or have recovered from HBV infection.

When the cancer cells from the primary sites in the colon escape into the bloodstream, the most likely location where they are lodged is the liver. Kelly et al. suggested that micro-metastasis occurred when cancer cells from the primary CRC escape from the primary location into the portal circulation ${ }^{27}$. The liver immune system removes the tumor cells at the beginning of metastasis ${ }^{28}$. However, in HBsAg-positive patients, the liver immune function was deficient. Peng et al. observed upregulation of the PD-1/PD-L1 pathway in patients with $\mathrm{CHB}$, thereby inhibiting the function and expression of interferon-r (INF-r) and CD8+ T cells, resulting in sustained liver cell damage ${ }^{29}$. Previous studies have suggested that the pathogenesis and metastasis of CRC are related to the upregulation of the PD-1/PD-L1 negative regulatory signaling pathway, which leads to immune escape ${ }^{30-32}$. Therefore, we believe that in $\mathrm{HBsAg}$ positive CRC patients, the liver immune function is impaired. Before surgery, when the primary tumor continues to enter the liver through blood flow, the consumption of the immune system in the liver is intensified, and the incidence of SYN-CRLM is increased. Similar phenomena were observed in primary liver cancer with HBV infection ${ }^{33,34}$.

When the primary tumor is removed surgically, the process of tumor cells continuously entering the liver through the blood is terminated. However, MET-CRLM is still present in about $15 \%$ of patients. This is because the liver's immune system is unable to effectively remove residual tumor cells. In this study, the incidence of MET-CRLM in group B was significantly lower than that of group A and C. It indicates that there may be some immune response related to HBV infection, which enhances the clearance of tumor cells in the liver.

A number of studies have pointed out that HBV related immune function has been enhanced in patients with HBV infection, and its antiviral function is better than that of patients with chronic hepatitis $B$ infection. Wu et al. noted that PD-1 expression in HBeAg-negative HBV patients was significantly lower than that in HBeAg-positive HBV patients ${ }^{35}$. Jung et al. found that the frequency of CD8+ CD45R0+ memory $T$ lymphocytes increased in acute HBV infection ${ }^{36}$. Penna showed that the frequency of HBVspecific T cells in self-limited acute hepatitis B was comparable to that observed in the acute stage of infection and, usually, was higher than that in patients with chronic HBV infection ${ }^{37}$. Zhang et al. pointed out that PD-1 expression was significantly upregulated on HBV-specific CD8 T cells in the early phase of acute HBV infection, and successful viral clearance correlated with a subsequent decrease in PD-1 expression ${ }^{38}$.

The results showed that HBsAg-positive HBV infection increased the risk of SYN-CRLM before surgery, probably by damaging hepatocytes and the liver immune system. HBsAg-negative HBV infection decreased the risk of MET-CRLM after surgery, probably by a good liver immune response. Compared to previous studies, our results can better explain the relationship between HBV and CRLM in the same cohort. However, this study is a single center retrospective analysis, and lack of HBV-DNA data. The 
occurrence of CRLM may be related to the primary tumor, HBV infection statues, and liver immunology, but the mechanism remain unknown. Therefore, we will carry out a multi-center prospective observational study in the future work, collect complete clinical and experimental data, and further study the mechanism of how HBV infection affects the occurrence of CRLM.

\section{Abbreviations}

CRC--colorectal cancer

CRLM--colorectal liver metastasis

SYN-CRLM--synchronous colorectal cancer liver metastasis

MET-CRLM--metachronous colorectal cancer liver metastasis

HBV--hepatitis B virus

HBVM--hepatitis B virus serological marker

HBsAg--hepatitis B virus surface antigen

anti-HBs--hepatitis B surface antibody

HBeAg--hepatitis B e antigen

anti-HBe--hepatitis B e antibody

anti-HBc--hepatitis B core antibody

CEA--carcinoembryonic antigen

CA19-9--carbohydrate antigen 19-9

AST--aspartate aminotransferase

ALT--alanine aminotransferase

FIB-4--fibrosis index based on the four factors

OS--overall survival

LDFS--liver disease-free survival

HR--hazard ratio

Cl--confidence interval 


\section{Declarations}

\section{Acknowledgements}

This work was supported by Guangzhou Science and Technology Programme Key Projects

(201604020005); Natural Science Foundation of Guangdong Province (No. 2017A030313580) and The Fundamental Research Funds for the Central Universities (Sun Yat-sen University), National Key Clinical Discipline (No. 17ykpy67)

\section{Author contributions}

Project administration: Huang Meijin and Wang Jianping

Writing - review \& editing: Huang Pinzhu; Huang Meijin and Wang Jianping

Data curation; Investigation: Tan Shuyun, Lin Rongwan, Zhan Huanmiao, Wu Xiaofeng, Peng Shaoyong, Li Tuoyang Huang Mingzhe and Xiao Yuanlv

Formal analysis; Software; Methodology; Writing- original draft: Zhou Jiaming, Huang Pinzhu and Guo Xiaoyan

Funding acquisition: Wang Jianping and Huang Pinzhu

\section{Competing interests}

The authors declare no competing interests.

\section{References}

1 Bray, F. et al., Global cancer statistics 2018: GLOBOCAN estimates of incidence and mortality worldwide for 36 cancers in 185 countries. CA Cancer J Clin68 394 (2018).

2 Chen, W. et al., Cancer incidence and mortality in China, 2014. Chin J Cancer Res30 1 (2018).

3 Adam, R., Colorectal cancer with synchronous liver metastases. BRIT J SURG94 129 (2007).

4 Rees, M., Tekkis, P. P., Welsh, F. K. S., O’Rourke, T. \& John, T. G., Evaluation of Long-term Survival After Hepatic Resection for Metastatic Colorectal Cancer. ANN SURG247 125 (2008).

$5 \quad$ Favoriti, P. et al., Worldwide burden of colorectal cancer: a review. Updates in Surgery68 7 (2016). 

Oncology16 3 (2007).

7 Manfredi, S. et al., Epidemiology and Management of Liver Metastases From Colorectal Cancer. ANN SURG244 254 (2006).

8 Trepo, C., Chan, H. L. \& Lok, A., Hepatitis B virus infection. LANCET384 2053 (2014).

9 Tanaka, M. et al., Hepatitis B and C Virus Infection and Hepatocellular Carcinoma in China: A Review of Epidemiology and Control Measures. J EPIDEMIOL21 401 (2011).

10 Luo, Z., Li, L. \& Ruan, B., Impact of the implementation of a vaccination strategy on hepatitis B virus infections in China over a 20-year period. INT J INFECT DIS16 e82 (2012).

11 Bréchot, C., Gozuacik, D., Murakami, Y. \& Paterlini-Bréchot, P., Molecular bases for the development of hepatitis B virus (HBV)-related hepatocellular carcinoma (HCC). SEMIN CANCER BIOL10 211 (2000).

12 Chuang, S., Vecchia, C. L. \& Boffetta, P., Liver cancer: Descriptive epidemiology and risk factors other than HBV and HCV infection. CANCER LETT286 9 (2009).

13 Levrero, M. \& Zucman-Rossi, J., Mechanisms of HBV-induced hepatocellular carcinoma. J HEPATOL64 S84 (2016).

14 Luo, G. et al., HBV infection increases the risk of pancreatic cancer: a meta-analysis. CANCER CAUSE CONTROL24 529 (2013).

15 Wei, X. L. et al., The status of HBV infection influences metastatic pattern and survival in Chinese patients with pancreatic cancer. J TRANSL MED11 249 (2013).

16 Zhou, X. et al., Both chronic HBV infection and naturally acquired HBV immunity confer increased risks of B-cell non-Hodgkin lymphoma. BMC CANCER19 (2019).

17 Utsunomiya, T. et al., Rare occurrence of colorectal cancer metastasis in livers infected with Hepatitis B or C virus. The American Journal of Surgery177 279 (1999).

18 Song, E., Chen, J., Ou, Q. \& Su, F., Rare occurrence of metastatic colorectal cancers in livers with replicative hepatitis B infection. AM J SURG181 529 (2001).

19 Augustin, G., Bruketa, T., Korolija, D. \& Milosevic, M., Lower incidence of hepatic metastases of colorectal cancer in patients with chronic liver diseases: meta-analysis. Hepatogastroenterology601164 (2013). 
Cai, B. et al., Patients with chronically diseased livers have lower incidence of colorectal liver metastases: a meta-analysis. PLOS ONE9 e108618 (2014).

21

Zhao, Y. et al., Hepatitis B Virus Infection Predicts Better Survival In Patients With Colorectal Liver-only Metastases Undergoing Liver Resection. J CANCER9 1560 (2018).

22

Huo, T. et al., Effect of Concomitant Positive Hepatitis B Surface Antigen on the Risk of Liver Metastasis: A Retrospective Clinical Study of 4033 Consecutive Cases of Newly Diagnosed Colorectal Cancer. CLIN INFECT DIS66 1948 (2018).

23

Kow, A. W. C., Hepatic metastasis from colorectal cancer. Journal of Gastrointestinal Oncology101274 (2019).

24 Qiu, H., HBV infection decreases risk of liver metastasis in patients with colorectal cancer: A cohort study. WORLD J GASTROENTERO17 804 (2011).

25 HCV hepatitis. ANN ITAL CHIR84 323 (2013).

26 Wang, F. S., Shao, Z. G., Zhang, J. L. \& Liu, Y. F., Colorectal liver metastases rarely occur in patients with chronic hepatitis virus infection. Hepatogastroenterology59 1390 (2012).

27 Kelly, R. J., Kemeny, N. E. \& Leonard, G. D., Current strategies using hepatic arterial infusion chemotherapy for the treatment of colorectal cancer. Clin Colorectal Cancer5 166 (2005).

28 Pancione, M. et al., Immune Escape Mechanisms in Colorectal Cancer Pathogenesis and Liver Metastasis. J IMMUNOL RES2014 1 (2014).

29 Peng, G. et al., PD-1 upregulation is associated with HBV-specific T cell dysfunction in chronic hepatitis B patients. MOL IMMUNOL45 963 (2008).

$30 \quad$ Kuai, W. et al., Prognostic Impact of PD-1 and Tim-3 Expression in Tumor Tissue in Stage I-III Colorectal Cancer. BIOMED RES INT2020 5294043 (2020).

31 Goltz, D., Gevensleben, H., Dietrich, J. \& Dietrich, D., PD-L1 (CD274) promoter methylation predicts survival in colorectal cancer patients. ONCOIMMUNOLOGY6 e1257454 (2017).

32 Chang, Y. et al., 494 PD1-positive tumor-infiltrating lymphocytes are associated with poor clinical outcome after pulmonary metastasectomy for colorectal cancer. J INVEST DERMATOL138 S84 (2018).

33

Liu, X. et al., PD-1+ TIGIT+ CD8+ T cells are associated with pathogenesis and progression of patients with hepatitis B virus-related hepatocellular carcinoma. Cancer Immunology, Immunotherapy68 
2041 (2019).

34

Li, Z. et al., Genetic polymorphisms of immune checkpoint proteins PD-1 and TIM-3 are associated with survival of patients with hepatitis B virus-related hepatocellular carcinoma. Oncotarget7 26168 (2016).

35

Wu, J. et al., Suppression of Furin by Interferon-y and the Impact on Hepatitis B Virus Antigen Biosynthesis in Human Hepatocytes. The American Journal of Pathology181 19 (2012).

36

Jung, M. C. et al., Increased frequency of CD8+ CD45R0+ memory T lymphocytes in acute hepatitis B virus infection. J HEPATOL18 295 (1993).

37

Penna, A. et al., Long-lasting memory T cell responses following self-limited acute hepatitis B. The Journal of clinical investigation98 1185 (1996).

38

Zhang, Z. et al., Dynamic Programmed Death 1 Expression by Virus-Specific CD8 T Cells Correlates With the Outcome of Acute Hepatitis B. GASTROENTEROLOGY134 1938 (2008).

\section{Tables}




\begin{tabular}{|c|c|c|}
\hline Factors & Number & Percentage (\%) \\
\hline \multicolumn{3}{|l|}{ Gender } \\
\hline Male & 1922 & 61.37 \\
\hline Female & 1210 & 38.63 \\
\hline \multicolumn{3}{|l|}{ Age (years old) } \\
\hline \multicolumn{3}{|c|}{ Median $56 \pm 11.17$, range from 17 to 95} \\
\hline \multicolumn{3}{|l|}{ Tumor Location } \\
\hline Rectum & 1526 & 48.72 \\
\hline Colon & 1606 & 51.28 \\
\hline \multicolumn{3}{|l|}{ HBV infection statues } \\
\hline HBsAg (+) infection (group A) & 413 & 13.19 \\
\hline HBsAg (-) infection (group B) & 638 & 20.37 \\
\hline Non-infection (group C) & 2081 & 66.44 \\
\hline \multicolumn{3}{|c|}{ Surgery for primary tumor resection } \\
\hline Presence & 2893 & 92.37 \\
\hline Absence & 239 & 7.63 \\
\hline \multicolumn{3}{|l|}{ CRLM* $^{*}$} \\
\hline SYN-CRLM & 425 & 13.57 \\
\hline MET-CRLM & 404 & 12.90 \\
\hline No CRLM & 2211 & 70.59 \\
\hline Lost during follow-up & 92 & 2.94 \\
\hline
\end{tabular}


Table 2. Difference between HBV infection groups

Group A Group B Group C

HBsAg(+) infection $\mathrm{HBsAg}(-)$ infection No infection

$N=413 \rrbracket \% \rrbracket \quad N=638 \rrbracket \% \square \quad N=2081 \rrbracket \% \rrbracket$

Gender

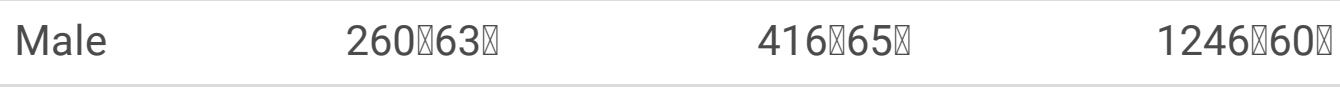

\begin{tabular}{|c|c|}
\hline Female & 153 \\
\hline
\end{tabular}

Age

$>=56$

$178 \rrbracket 43 \rrbracket$

358₫56》

$<0.01$

$<56$

235区57囚

$280 \rrbracket 44 \rrbracket$

1131囚54囚

Location

(2)

Rectum
Colon
Differentiation

\begin{tabular}{ll} 
High or middle & $353 \rrbracket 85 \rrbracket$ \\
\hline Low & $60 \rrbracket 15 \rrbracket$ \\
\hline T stage & \\
\hline
\end{tabular}

$571 \llbracket 89 \rrbracket$

$329852 \rrbracket$

0.12

$67 \rrbracket 11 \rrbracket$

1011ه49凶

$309 \llbracket 48 \rrbracket$

1070ه51》

3

3 or 4

$\mathrm{N}$ stage

300ه80》

452ख76》

145囚24】

0.08

1 or 2

74『20》

$145 \triangle 24 \bowtie$

0

M stage

1

0

CRLM

I

SYN-CRLM

MET-CRLM

No CRLM

\begin{tabular}{l}
$165 \rrbracket 44 \rrbracket$ \\
\hline $209 \llbracket 56 \rrbracket$ \\
\hline $148 \rrbracket 36 \rrbracket$ \\
$265864 \rrbracket$
\end{tabular}

\begin{tabular}{ll}
\hline $234 \rrbracket 39 \rrbracket$ & $794 \rrbracket 41 \rrbracket$ \\
\hline $363 \rrbracket 61 \rrbracket$ & $1128 \rrbracket 59 \rrbracket$
\end{tabular}

0.32

1797凶86》

284冈14】

0.23

1469ه76】

453囚24】

\begin{tabular}{|c|c|}
\hline $152 \rrbracket 24 \rrbracket$ & 641ه31ه \\
\hline 486ه76ه & 1440ه69ه \\
\hline & 0.01 \\
\hline 74ه12】 & $281 \rrbracket 14 \rrbracket$ \\
\hline 64ه10ه & $286 \rrbracket 14 \rrbracket$ \\
\hline 491ه78囚 & 1447刃72》 \\
\hline
\end{tabular}




\begin{tabular}{|c|c|c|c|c|}
\hline CEA & & & & 0.29 \\
\hline$>5 \mathrm{ng} / \mathrm{ml}$ & 140ه34】 & 195®31》 & $624 \rrbracket 30 \rrbracket$ & \\
\hline$<=5 \mathrm{ng} / \mathrm{ml}$ & $273 \rrbracket 66 \rrbracket$ & 443囚69』 & 1457ه70ه & \\
\hline CA19-9 & & & & 0.74 \\
\hline ه37U/ml & 77ه19ه & 107凶17》 & $365 \rrbracket 18 \rrbracket$ & \\
\hline$<=37 \mathrm{U} / \mathrm{ml}$ & $336 \rrbracket 81 \rrbracket$ & $531 \rrbracket 83 \rrbracket$ & 1716ه82》 & \\
\hline AST & & & & $<0.01$ \\
\hline$\otimes 40 \mathrm{U} / \mathrm{L}$ & $337 \rrbracket 82 \rrbracket$ & $130 \rrbracket 20 \rrbracket$ & $454 \rrbracket 22 \rrbracket$ & \\
\hline$<=40 \mathrm{U} / \mathrm{L}$ & 76ه18ه & $508 \rrbracket 80 \rrbracket$ & 1627ه78ه & \\
\hline ALT & & & & $<0.01$ \\
\hline$>40 \mathrm{U} / \mathrm{L}$ & $267 \rrbracket 65 \rrbracket$ & $126 \rrbracket 20 \rrbracket$ & $427 \rrbracket 21 \rrbracket$ & \\
\hline$<=40 \mathrm{U} / \mathrm{L}$ & 146ه35》 & $512 \rrbracket 80 \rrbracket$ & 1654ه79ه & \\
\hline FIB-4 index & & & & $<0.01$ \\
\hline$>3.25$ & 119®29ه & 104囚16】 & $336 \rrbracket 16 \rrbracket$ & \\
\hline$<=3.25$ & 294『71》 & $534 \rrbracket 84 \rrbracket$ & $1745 \rrbracket 84 \rrbracket$ & \\
\hline
\end{tabular}

\begin{tabular}{|llll|}
\hline \multicolumn{4}{|c|}{ Table 3. Incidence rate of SYN-CRLM in different HBV infection statues N=3132 } \\
\hline SYN-CRLM & No SYN-CRLM (\%) & Total \\
\hline Group A & $70(16.9 \%)$ & $343(83.1 \%)$ & 413 \\
\hline Group B & $74(11.6 \%)$ & $564(88.4 \%)$ & 638 \\
\hline Group C & $281(13.5 \%)$ & $1800(86.5 \%)$ & 2081 \\
\hline P=0.05 & & & \\
\hline
\end{tabular}




\begin{tabular}{|llll|}
\hline \multicolumn{4}{|c|}{ Table 4. Incidence rate of MET-CRLM in different HBV infection statues N=2615 } \\
\hline & MET-CRLM & No MET-CRLM & Total \\
\hline Group A & $54(16.5 \%)$ & $273(83.5 \%)$ & 327 \\
\hline Group B & $64(11.5 \%)$ & $491(88.5 \%)$ & 555 \\
\hline Group C & $286(16.5 \%)$ & $1447(83.5 \%)$ & 1733 \\
\hline $\mathrm{P}=0.02$ & & & \\
\hline
\end{tabular}


Table 5. Risk factors of SYN-CRLM N=3132

Factor SYN-CRLM No SYN-CRLM Univariate Multivariate $\mathrm{HR} \quad 95 \% \mathrm{Cl}$ $\mathrm{N}=425(\%) \quad \mathrm{N}=2707(\%)$

Gender

0.71

Male

$257(60) \quad 1665(62)$

Female

$168(40) \quad 1042(38)$

Age

0.08

0.13

0.81

0.61-1.07

$>=56$

209 (49) $\quad 1458$ (54)

$<56$

$216(51)$

$1249(46)$

Location

0.02

0.28

0.86

$0.65-1.13$

Rectum

$185(44)$

1341 (50)

Colon

$240(56)$

$1366(50)$

Differentiation

$<0.01$

0.01

0.64

$0.45-0.89$

High or middle $312(73) \quad 2409(89)$

Low $\quad 113(27) \quad 298(11)$

T stage

$<0.01$

$<0.01$

4.04

2.12-7.67

3 or 4

267 (96) $1954(75)$

0,1 or 2

$11(4)$

$661(25)$

$\mathrm{N}$ stage

$<0.01$

$<0.01$

1.82

$1.36-2.43$

1 or 2

$186(67)$

1007 (39)

0

$92(33)$

1608 (61)

$M$ stage $<0.01$

$<0.01$

22.4

$16.1-35.2$

1

$425(100) \quad 470(17)$

0

$0(0)$

2237 (83)

HBV infection

$0.05<0.01$

group A

70 (16)

$343(13)$

Ref

group B

74 (17)

564 (21)

$<0.01$

0.19

$0.11-0.34$

group C

281 (67)

$1800(66)$

$<0.01$

0.29

0.18-0.47

CEA

$<0.01$

$<0.01$

3.91

2.88-5.30 


\begin{tabular}{|c|c|c|c|c|c|c|}
\hline$>5 \mathrm{ng} / \mathrm{ml}$ & $304(72)$ & 655 (24) & & & & \\
\hline$<=5 \mathrm{ng} / \mathrm{ml}$ & $121(28)$ & $2052(76)$ & & & & \\
\hline CA19-9 & & & $<0.01$ & $<0.01$ & 2.17 & $1.59-2.98$ \\
\hline ه37U/ml & $211(50)$ & $338(12)$ & & & & \\
\hline$<=37 \mathrm{U} / \mathrm{ml}$ & $214(50)$ & 2369 (88) & & & & \\
\hline AST & & & $<0.01$ & $<0.01$ & 0.277 & $0.18-0.42$ \\
\hline$\nabla 40 \mathrm{U} / \mathrm{L}$ & $85(20)$ & $836(31)$ & & & & \\
\hline$<=40 \mathrm{U} / \mathrm{L}$ & $340(80)$ & 1871 (69) & & & & \\
\hline ALT & & & $<0.01$ & $<0.01$ & 0.49 & $0.35-0.72$ \\
\hline$>40 \mathrm{U} / \mathrm{L}$ & $83(20)$ & 737 (27) & & & & \\
\hline$<=40 \mathrm{U} / \mathrm{L}$ & 342 (80) & 1970 (73) & & & & \\
\hline FIB-4 index & & & 0.45 & & & \\
\hline$>3.25$ & $70(16)$ & 489 (18) & & & & \\
\hline$<=3.25$ & $355(84)$ & 2218 (82) & & & & \\
\hline
\end{tabular}


Table 6. Risk factors of MET-CRLM N=2615

\begin{tabular}{|c|c|c|c|c|c|c|}
\hline Factors & $\begin{array}{l}\text { MET-CRLM } \\
\mathrm{N}=404(\%)\end{array}$ & $\begin{array}{l}\text { No MET-CRLM } \\
\text { N=2211(\%) }\end{array}$ & Univariate & Multivariate & $\mathrm{HR}$ & $95 \% \mathrm{Cl}$ \\
\hline Gender & & & 0.08 & 0.15 & 1.16 & $0.95-1.43$ \\
\hline Male & $266(66)$ & $1351(61)$ & & & & \\
\hline Female & $138(34)$ & 860 (39) & & & & \\
\hline Age & & & 0.44 & & & \\
\hline$>=56$ & $210(52)$ & $1195(54)$ & & & & \\
\hline$<56$ & $194(48)$ & $1016(46)$ & & & & \\
\hline Location & & & $<0.01$ & 0.48 & 0.93 & $0.76-1.14$ \\
\hline Rectum & $169(42)$ & $1141(52)$ & & & & \\
\hline Colon & $235(58)$ & $1070(48)$ & & & & \\
\hline Differentiation & & & $<0.01$ & 0.04 & 0.77 & $0.61-0.99$ \\
\hline High or middle & 319 (79) & 2017 (91) & & & & \\
\hline Low & $85(21)$ & $194(9)$ & & & & \\
\hline T stage & & & $<0.01$ & 0.05 & 1.54 & $1.01-2.36$ \\
\hline 3 or 4 & $378(94)$ & $1576(71)$ & & & & \\
\hline 0,1 or 2 & $26(6)$ & $635(29)$ & & & & \\
\hline $\mathrm{N}$ stage & & & $<0.01$ & 0.60 & 1.06 & $0.85-1.32$ \\
\hline 1 or 2 & $263(65)$ & $744(34)$ & & & & \\
\hline 0 & $141(35)$ & $1467(66)$ & & & & \\
\hline M stage & & & $<0.01$ & $<0.01$ & 10.12 & $7.90-12.95$ \\
\hline 0 & $124(31)$ & $2062(93)$ & & & & \\
\hline 1 & $280(69)$ & $149(7)$ & & & & \\
\hline HBV infection & & & 0.02 & 0.01 & & \\
\hline group A & $54(13)$ & $273(12)$ & & 0.04 & 1.47 & $1.02-2.10$ \\
\hline group B & $64(16)$ & $491(22)$ & & Ref & - & - \\
\hline group C & $286(71)$ & $1447(65)$ & & $<0.01$ & 1.49 & $1.14-1.95$ \\
\hline CEA & & & $<0.01$ & $<0.01$ & 1.90 & $1.52-2.38$ \\
\hline
\end{tabular}




\begin{tabular}{|c|c|c|c|c|c|c|}
\hline$>5 \mathrm{ng} / \mathrm{ml}$ & $223(55)$ & 399 (18) & & & & \\
\hline$<=5 \mathrm{ng} / \mathrm{ml}$ & $181(45)$ & $1812(82)$ & & & & \\
\hline CA19-9 & & & $<0.01$ & $<0.01$ & 1.50 & $1.19-1.89$ \\
\hline ه37U/ml & 135 (33) & $182(8)$ & & & & \\
\hline$<=37 \mathrm{U} / \mathrm{ml}$ & 269 (67) & $2029(92)$ & & & & \\
\hline AST & & & 1.00 & & & \\
\hline$\nabla 40 \mathrm{U} / \mathrm{L}$ & $124(31)$ & $682(31)$ & & & & \\
\hline$<=40 \mathrm{U} / \mathrm{L}$ & $280(69)$ & 1529 (69) & & & & \\
\hline ALT & & & 0.58 & & & \\
\hline$>40 \mathrm{U} / \mathrm{L}$ & $104(26)$ & $601(27)$ & & & & \\
\hline$<=40 \mathrm{U} / \mathrm{L}$ & $300(74)$ & $1610(73)$ & & & & \\
\hline FIB-4 index & & & 0.18 & & & \\
\hline$>3.25$ & $83(21)$ & 392 (18) & & & & \\
\hline$<=3.25$ & $321(79)$ & 1819 (82) & & & & \\
\hline Chemotherapy & & & $<0.01$ & $<0.01$ & 3.11 & $1.91-5.04$ \\
\hline Presence & 382 (95) & $1280(58)$ & & & & \\
\hline Absence & $22(5)$ & $931(42)$ & & & & \\
\hline
\end{tabular}

Figures 


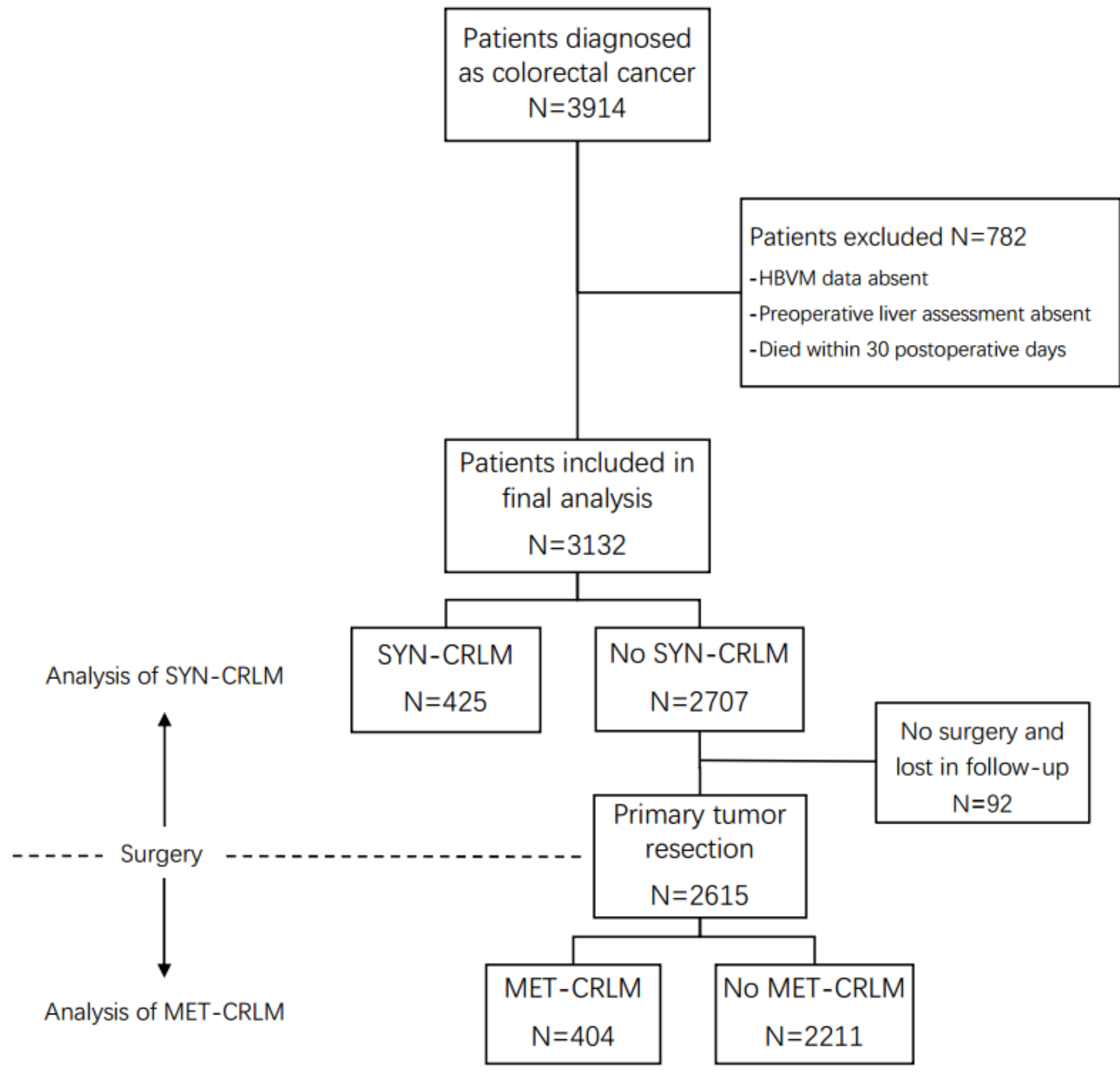

Figure 1

The flow chart of this study 


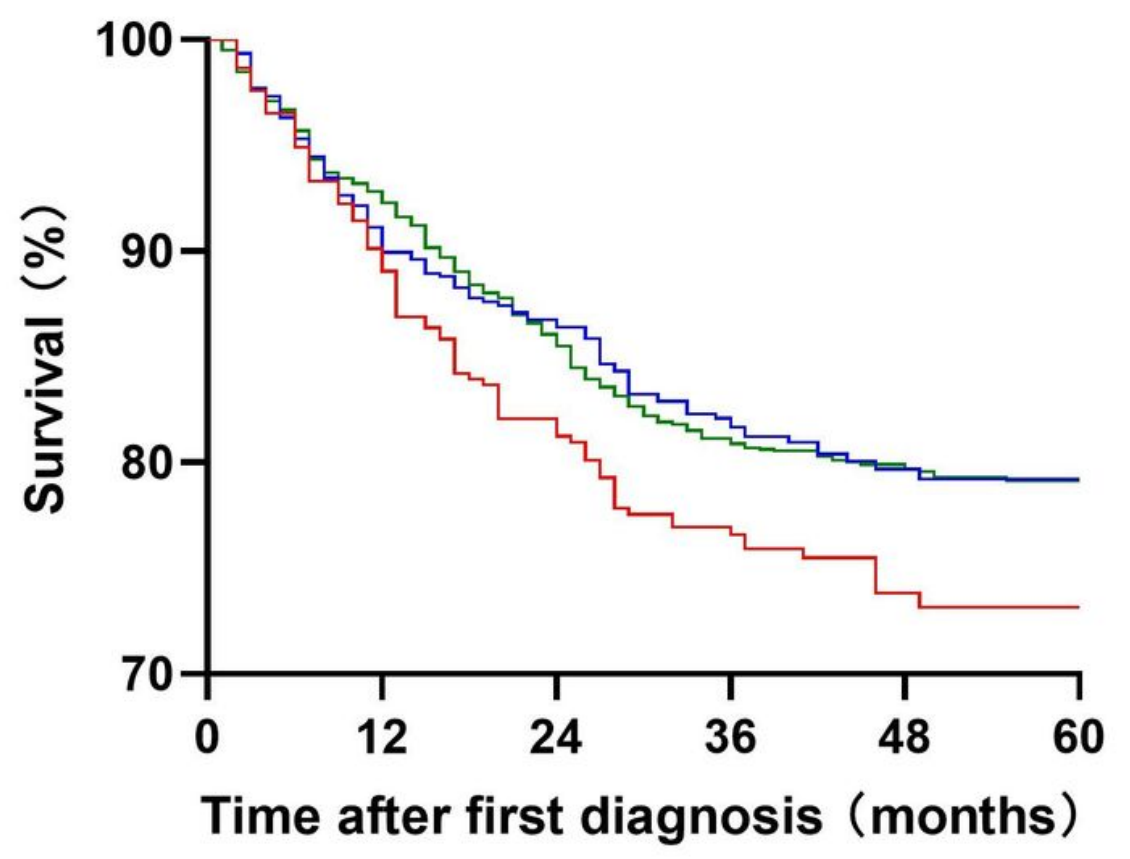

- HBsAg(+) infection

- HBsAg(-) infection

- Non-infection

$P$ value $=0.05$

Figure 2

The 5-year OS among different HBV infection groups $\mathrm{N}=2893$

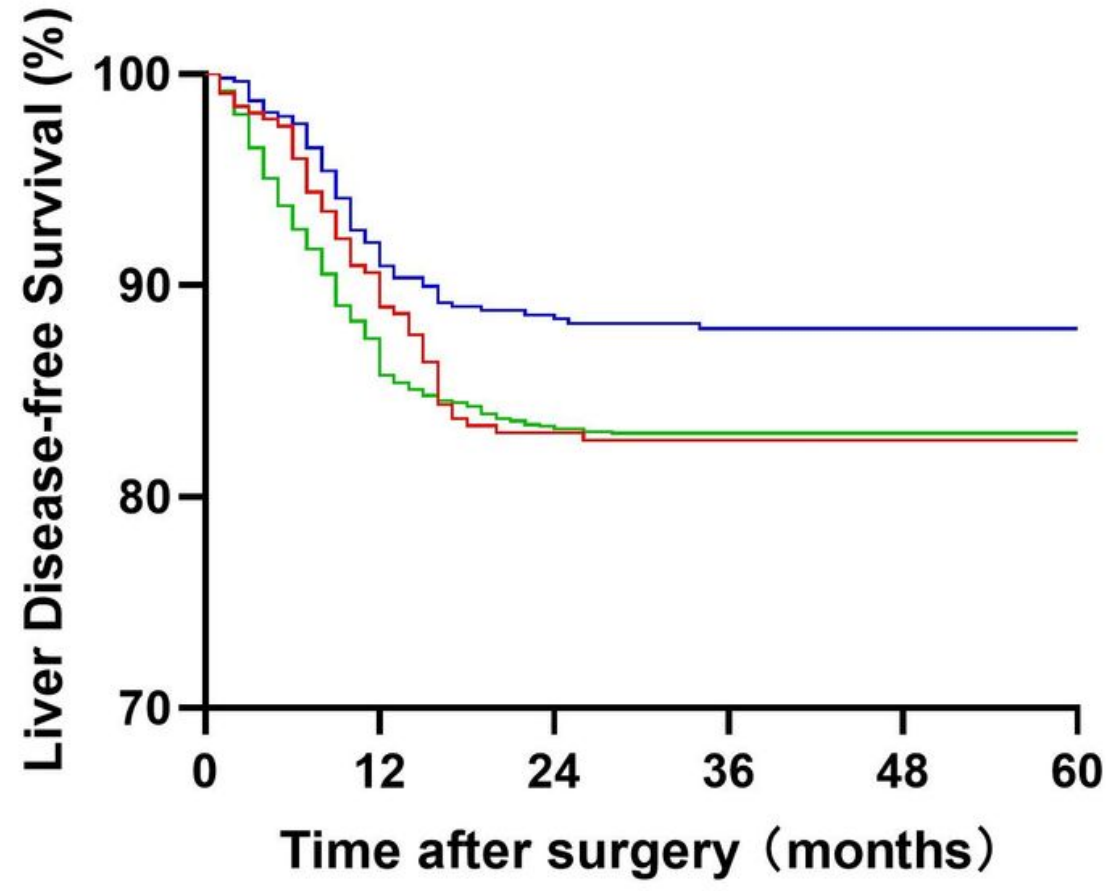

- HBsAg (+) infection

- HBsAg(-) infection

- Non-infection

$P$ value $=0.01$

Figure 3

The 5-year LDFS among different HBV infection groups $\mathrm{N}=2615$ 


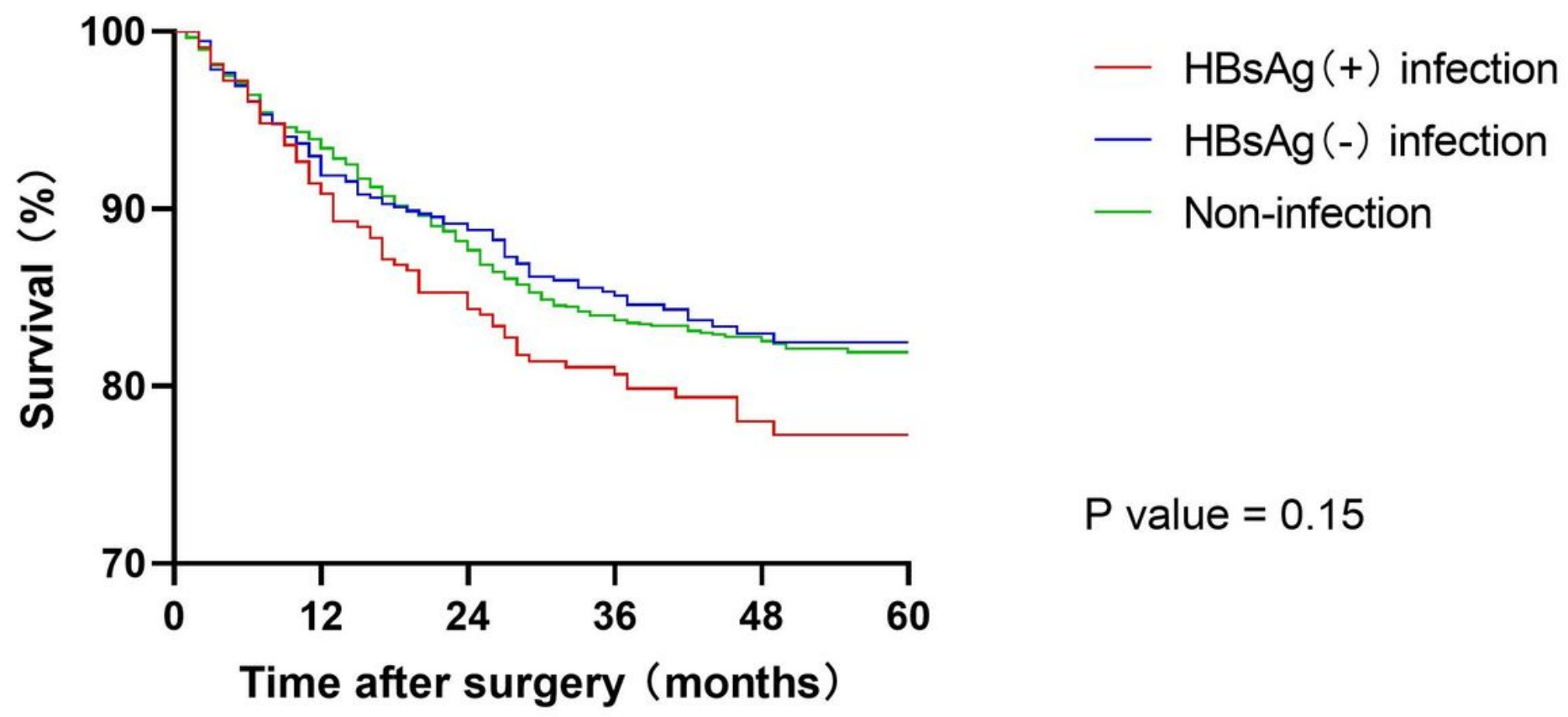

Figure 4

The 5-year OS among different HBV infection groups N=2615

\section{Supplementary Files}

This is a list of supplementary files associated with this preprint. Click to download.

- HBVCRLM3132supplementarytables20210228.docx 